\title{
Annette von Stockhausen \\ Der Brief der Synode von Ankyra 358 (Dok. 55)
}

\section{Einleitung}

Epiphanius von Salamis überliefert im 73. Kapitel seines Panarion den Brief einer Synode, die im Jahr 358 im kleinasiatischen Ankyra stattgefunden hat. Da dieser Brief gemeinhin als »Gründungsurkunde« der theologischen Gruppe der »Homöusianer « gilt, war er schon häufiger Gegenstand von Untersuchungen, wobei der Fokus in erster Linie auf dem theologischen Profil des Briefes lag. ${ }^{1}$

Der Brief der Synode von Ankyra ist daher selbstverständlich in die Sammlung der »Dokumente zum arianischen Streit« (Lfg. 4) aufgenommen worden, und zwar als Dok. 55. ${ }^{2}$ Im Zuge der Beschäftigung mit diesem Text wurde jedoch schnell deutlich, dass über den schlechten Überlieferungszustand und die damit verbundenen philologischen und editorischen Fragen hinausgehend, ${ }^{3}$ die uns bei allen bei Epiphanius überlieferten Texten große Kopfschmerzen bereitet haben, der Text auch sonst viel problematischer ist als bisher gedacht.

Relativ klar - wenn auch damit noch lange nicht immer einfach bzw. überhaupt zu lösen - ist die überlieferungs- und textkritische Problematik des Synodalschreibens:

Der Text des Panarion des Epiphanius ist für einen großen Teil der Schrift ${ }^{4}$ und darunter eben auch für das 73. Kapitel über die Homöusianer nur in einer einzigen Handschrift überliefert, dem heute in der Thüringischen Universitäts- und Landesbibliothek aufbewahrten Codex Ms. Bos. f. 1 aus dem Jahr 1304. ${ }^{5}$ Ist schon die Überlieferung eines

1 Einschlägige Literatur: Gummerus, Die homöusianische Partei, 66-89; Kopecek, A history of NeoArianism, 155-172; Steenson, Basil of Ancyra, 126-208; Lienhard, The Epistle of the Synod of Ancyra 358; Löhr, Entstehung der homöischen und homöusianischen Kirchenparteien, 63-75; Hanson, The Search for the Christian Doctrine, 350-357; Löhr, A Sense of Tradition; zuletzt Fairbairn, The Synod of Ancyra (358).

2 Der Band ist inzwischen erschienen: Hanns Christof Brennecke/Annette von Stockhausen/Christian Müller/Uta Heil/Angelika Wintjes, Athanasius Werke. Band III. Teil 1: Dokumente zur Geschichte des Arianischen Streits 318-328. 4. Lfg.: Bis zum Tomus ad Antiochenos (362), Berlin/Boston, 2014 (im Folgenden beziehen sich Verweise mit »Dok. « auf diesen Band); die hier vorgetragenen Überlegungen sind in diese Edition eingegangen.

3 Die schlechte Überlieferung wird natürlich in der gesamten bisherigen Literatur erwähnt, allerdings - abgesehen von Gummerus, Die homöusianische Partei (aber auch da nicht im nötigen Maße) - ohne daraus Konsequenzen zu ziehen.

4 Kap. 65-80; d. h. in der GCS-Edition für den gesamten 3. Band (Holl, Epiphanius. Dritter Band).

5 Zur Handschrift vgl. Stockhausen, Katalog der griechischen Handschriften, 685-689; für die Textüberlieferung und die stemmatische Einordnung des Textes ist nach wie vor Holl, Die handschriftliche Überlieferung des Epiphanius, 76-87 einschlägig. 
Textes durch eine einzige Handschrift an sich ungünstig, so erweist sich dieser Umstand im Falle des Epiphanius als umso problematischer, als der Codex einen oftmals verstümmelten und fehlerhaften Text bietet; dieser verstümmelte und fehlerhafte Text geht dabei mit größter Wahrscheinlichkeit nicht auf den (unbekannten) Schreiber der Handschrift selbst, sondern mindestens auf seine Vorlage oder wahrscheinlich sogar noch weiter zurück.

In der Spätantike und im Mittelalter wurde der Text des Epiphanius offensichtlich nur sehr wenig gelesen und rezipiert, im byzantinischen Bereich war er auch spätes-

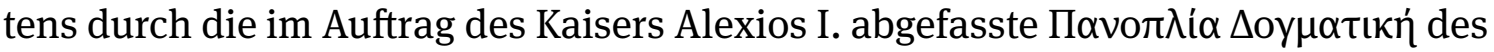
Euthymios Zigabenos überholt. ${ }^{6}$ Bemühungen um den Text setzen erst im Zeitalter der Reformation wieder ein, als Philipp Melanchthon sich die Handschrift vom Erfurter Reformator Johannes Lang auslieh (und dabei zum Teil auch in margine kommentierte $^{7}$ ) und sie schließlich an den Basler Drucker Johannes Oporinus weitergab. In Basel wurde aus ihr zunächst durch Janus Cornarius eine lateinische Übersetzung angefertigt, die auch zuerst (1543) gedruckt wurde, ${ }^{8}$ ehe dann 1544 schließlich der von Oporinus herausgegebene griechische Druck erscheinen konnte. ${ }^{9}$ Die Edition von Oporinus zeichnet sich dabei vor allem dadurch aus, dass er den Text des Epiphanius sehr treu nach der Handschrift abdruckt, eine textkritische Arbeit fehlt hier vollständig. Erste wichtige textkritische Überlegungen (und Emendationen) gehen dann auf den Jesuiten Dionysius Petavius (und damit ins 17. Jh.) zurück. ${ }^{10}$ Den bisherigen Meilenstein für die Epiphanius-Edition bildet sicherlich die Edition von Karl Holl in den »Griechischen christlichen Schriftstellern «; ${ }^{11}$ mehr als alle seine Vorgänger griff Holl in den meist zurecht als verderbt identifizierten Text ein und schlug oft wortreiche und weitreichende Emendationen vor.

Holls Edition stellte daher zunächst die Grundlage für die Edition in den »Athanasius Werken « dar; allerdings stellte sich sehr schnell heraus, dass Holls Edition keinesfalls ohne weiteres übernommen werden kann, sondern die vielen überflüssigen Emendationen ${ }^{12}$ und die zum Zeil weit über das Ziel hinausschießenden Ergänzungen ${ }^{13}$ der (meist zurecht) angenommenen Lücken korrigiert werden müssen. Im weiteren

6 PG 130,20-1360; eine vollständige kritische Edition liegt bisher nicht vor.

7 Ediert in Dummer/Markschies, Philipp Melanchthons Randbemerkungen.

8 Cornarius, Epiphanii Contra octoaginta haereses opus (= VD16 E 1644).

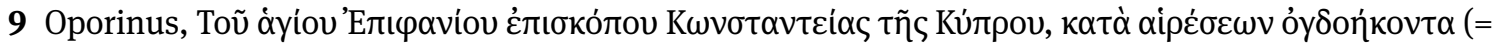
VD16 E 1643).

10 Petavius, Epiphanii Opera omnia in duos tomos distributa, nachgedruckt in Mignes Patrologia graeca 41 und 42.

11 Für das Schreiben der Synode von Ankyra Holl, Epiphanius. Dritter Band, 268,30-284,10 (die von Holl/Dummer bearbeitete zweite Auflage trägt für unseren Text nur einige neuere Editionen nach), daneben sind weiterhin die Konjekturen von Gummerus, Die homöusianische Partei wichtig.

12 V. a. grammatikalisch nicht notwendige Korrekturen, die nur einen stilistisch besseren Text ergeben.

13 Um Holl gerecht zu werden, muss natürlich gesagt werden, dass er diese meist nur im Apparat verzeichnet und nicht in den Text übernommen hat (im Apparat der Athanasius Werke sind solche 
Verlauf der Arbeit stellten sich darüber hinaus Unstimmigkeiten in der Wiedergabe früherer Konjekturen (v. a. von Petavius) im Vergleich mit den früheren Editionen heraus, so dass die Handschrift noch einmal kollationiert wurde und dabei auch einige Fehler Holls beim Lesen der Handschrift korrigiert werden konnten. ${ }^{14}$

Bei der sehr intensiven Beschäftigung mit dem Text der Synode von Ankyra stellte sich aber vor allem heraus, dass er auch jenseits der textkritischen Fragen im engeren Sinne, was seinen Aufbau und damit zusammenhängend seine Überlieferungsgeschichte angeht, erklärungsbedürftiger ist als bisher gesehen wurde. Um diese eigentliche Problematik des Textes zu erfassen, ist jedoch zunächst ein Ausholen in zweierlei Hinsicht nötig, nämlich zum einen durch eine neuerliche Betrachtung des Kontextes des Textes im Panarion des Epiphanius., zum anderen durch einen vergleichenden Blick auf andere zeitgenössische Synodalschreiben. In einem weiteren Schritt möchte ich dann auf den Aufbau des Briefes und auf hier zu beobachtende Eigenheiten des Textes eingehen, die eventuell Rückschlüsse auf seine Entstehung ermöglichen. Dadurch kann schließlich auch die Synode von Ankyra selbst, über die wir sonst nur sehr spärlich aus den Kirchenhistorikern Informationen haben, ${ }^{15}$ vielleicht in einem etwas anderen und klareren Licht erscheinen.

Ihre Geschichte stellt sich wie folgt dar: ${ }^{16}$ Basilius von Ankyra hatte (nach dem Zeugnis des Sozomenus ${ }^{17}$ aus Anlass einer Kirchweihe) vor dem Osterfest $358^{18}$, d. h. wahrscheinlich in der Fastenzeit des Jahres 358, zwölf benachbarte Bischöfe (vgl. die Unterschriften in $\S 27^{19}$ ) zu einer Synode nach Ankyra eingeladen, auf der neben weiteren Zeugenaussagen der Beschwerdebrief Georgs von Laodizäa über die Unterstützung des Aëtius und seiner Schüler seitens des neuen antiochenischen Bischofs Eudoxius (Dok. 54) verlesen und Eudoxius wohl auch verurteilt wurde. ${ }^{20}$ Die Synodalen entwarfen daraufhin ein Synodalschreiben, das auch eine ausführliche theologische Erklärung enthält, die sowohl gegen »Sabellianismus « als auch (ohne die Gegner beim Namen zu nennen) gegen »anhomöische« Thesen Stellung bezieht und als erstes Dokument der

Fälle durch »susp. « im Gegensatz zu »coni. « gekennzeichnet, vgl. z. B. den Apparat auf S. 390); im Text ist bei Holl an der jeweiligen Stelle die Lücke nur durch ein Sternchen gekennzeichnet.

14 Die Lesefehler liegen wahrscheinlich nicht zuletzt darin begründet, dass sich die Handschrift vor allem auf Grund von Tintenfraß bis zum Jahr 1974, als sie einer grundlegenden Restaurierung unterzogen wurde, in einem sehr schlechten Zustand befand und zum Teil praktisch nicht mehr lesbar war, wie eine Mikroverfilmung der Handschrift durch die Thüringer Universitäts- und Landesbibliothek zeigt, die vor der Restaurierung hergestellt wurde. Es ist zu vermuten, dass Holl zumindest gelegentlich eher auf die Editio princeps als auf die Handschrift selbst zurückgegriffen hat.

15 Soz., h. e. IV 13; Philost., h. e. IV 8; Thdt., h. e. II 25.

16 Vgl. jetzt auch AW III 4, Dok. 55 Einleitung.

17 Soz., h.e. IV 13,1.

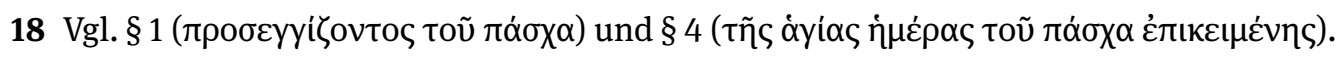

19 Die Bischöfe sind größtenteils unbekannt.

20 Vgl. die bereits oben genannte Stelle Soz., h. e. IV 13,1 sowie Philost., h. e. IV 8, Thdt., h. e. II 25 und Dok. 56.4. 
Gruppe der »Homöusianer « gilt. Die Synode sandte anschließend eine Delegation ${ }^{21}$ bestehend aus Basilius von Ankyra und Eustathius von Sebaste, denen sich dann noch der auf der Synode nicht anwesende Eleusius von Kyzikos und ein Presbyter namens Leontius anschlossen, zum Kaiser nach Sirmium, damit dieser gegen Eudoxius von Antiochien vorginge. ${ }^{22}$

\section{Das Schreiben im Kontext des Panarion des Epiphanius}

In einem zweiten Schritt soll nun das Schreiben der Synode von Ankyra im Kontext des Panarion des Epiphanius von Salamis betrachtet werden: ${ }^{23}$

Das Schreiben findet sich in Kapitel 73 des Panarion. Dieses Kapitel gehört zum ersten Band des dritten Buches und behandelt nach Audianern (Kap. 70), Photinianern

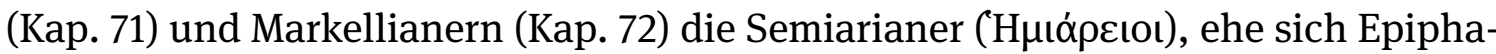
nius mit Pneumatomachen (Kap. 74), Aërianern (Kap. 75) und abschließend mit den Anhomöern (Kap. 76) beschäftigt.

Im Kapitel über die Semiarianer ordnet Epiphanius diese (wie üblich) zunächst häresiologisch ein. ${ }^{24}$ In der Einleitung nennt er dann Basilius von Ankyra sowie Georg von Laodizäa als ihre Protagonisten, beschreibt ihre theologische Position mit dem

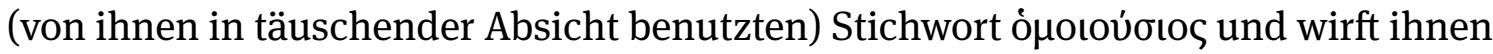
schließlich auch pneumatomachische Positionen vor (§ 1) - der Blick des Epiphanius aus den späten 70er Jahren auf die früheren Ereignisse wird hier (wie auch bei der noch folgenden Einordnung des Kyrill von Jerusalem oder vor allem des Meletius) ganz deutlich. Zur Bekräftigung seiner häresiologischen Einordnung will Epiphanius anschließend zwei Texte zitieren. ${ }^{25}$ Der erste zitierte Text (§ 2-11) ist das Schreiben der Synode von Ankyra (Dok. 55), der zweite (§ 12-22) der Traktat des Georg von Laodizäa

21 Vgl. Soz., h.e. IV 13,5.

$22 \mathrm{Zu}$ den Ereignissen in Sirmium vgl. Dok. 56.

23 Das große antihäretische Werk des Epiphanius wird meist nur als Steinbruch für dogmengeschichtliche Untersuchungen (sei es zur Gnosis oder zu den Häresien des 4. Jahrhunderts) verwendet. Erst in jüngerer Zeit ist Epiphanius vermehrt als Autor in den Blick genommen worden, vgl. jetzt Kim, Epiphanius of Cyprus und Jacobs, Epiphanius of Cyprus mit Überblick über den Forschungsstand.

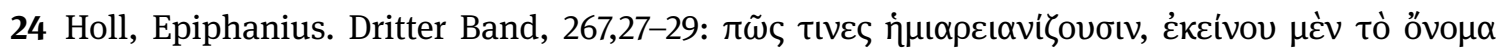

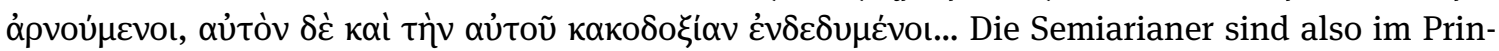
zip Arianer, die dies aber leugnen.

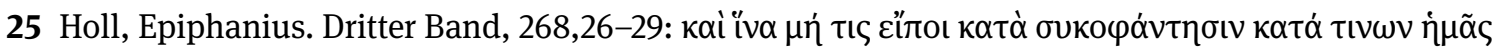

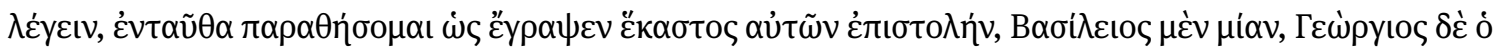

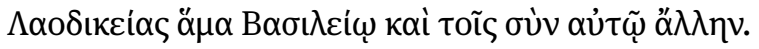


und des Basilius von Ankyra (Dok. 58). ${ }^{26}$ Nach den Briefen (§ 23) steht das Fazit, dass der zu Beginn aufgestellte Vorwurf sich anhand der zitierten Texte als zutreffend erwiesen hat. ${ }^{27}$ An die Dokumente der Homöusianer anschließend setzt Epiphanius seine häresiologische Darstellung mit dem Bericht über eine (nicht theologisch begründete, sondern auf persönlichen Animositäten beruhende) Spaltung der Semiarianer fort (§ 24) und zitiert nach einer ähnlichen Einleitung wie zu Beginn als nächsten Beweistext (§ 25) aus dem Kontext der Synode von Seleucia 359 die theologische Erklärung der Gruppe um Acacius von Caesarea mit ihrer Unterschriftenliste (Dok. 60.2). Wiederum folgt ein Fazit mit einer (an den oben eingeführten Gedanken der Spaltungen anknüpfenden) Überleitung (§ 27 f.) zum letzten Beweistext (§ 29-33), die im Kontext der Synode von Antiochien $360 \mathrm{zu}$ verortende (homöische) Predigt des Meletius (Dok. 65), an die Epiphanius das oben bereits erwähnte wertschätzende Urteil über Meletius anfügt (§ 34 f.). Am Ende des Kapitels (§ 36) steht eine Sermocinatio mit einer Widerlegung der "semiarianischen" Theologie und ein abschließendes Fazit (§ 37).

Für unsere Fragestellung sind nur die beiden zusammen zitierten homöusianischen Dokumente von Interesse. Bei beiden ist von redaktionellen Eingriffen des Epiphanius auszugehen, dazu auch von Textverlusten im Laufe der Überlieferung des EpiphaniusTextes.

Die Überlieferung dieser beiden Dokumente ist lückenhaft: Beim Brief der Synode von Ankyra fehlt, wie schon erwähnt, ${ }^{28}$ auf jeden Fall der Brief Georgs (Dok. 54) im Anhang, und auch die Unterschriftenliste ist nur summarisch wiedergegeben. ${ }^{29}$ Auch die Überlieferung des Traktates des Georg von Laodizäa und des Basilius von Ankyra (Dok. 58) ist problematisch: ${ }^{30}$ Der briefliche Rahmen ist (redaktionell) entfernt, vielleicht fehlt auch die Einleitung sowie (eher unwahrscheinlich ${ }^{31}$ ) der Schluss nach den als Beweis zitierten Dokumenten $56.5^{32}$ und $57.3^{33}$. Ein schwerwiegendes textliches Pro-

26 Wahrscheinlich hat Epiphanius fälschlicherweise diesen zweiten Text für den Brief gehalten, von dem in Dok. 55,4 gesagt wird, dass sich im Anhang des Briefes der Synode eine Abschrift eines Briefes Georgs von Laodizäa befindet. Epiphanius überliefert ihn aber nicht, sondern er ist mit dem bei Soz., h. e. IV 13,2 f. überlieferten Brief (Dok. 54) zu identifizieren.

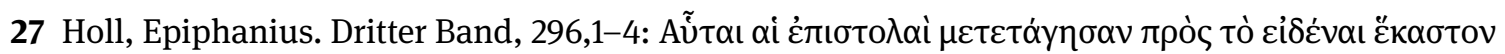

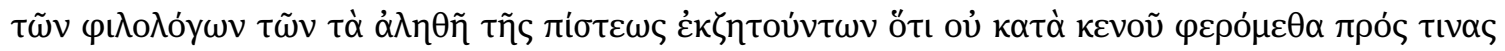

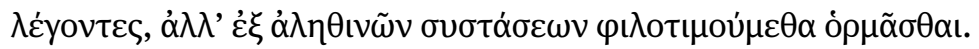

$28 \mathrm{Vgl}$. Anm. 26.

29 Vgl. unten S. 199.

30 Vgl. auch die Einleitung in AW III 3, $426 \mathrm{f}$.

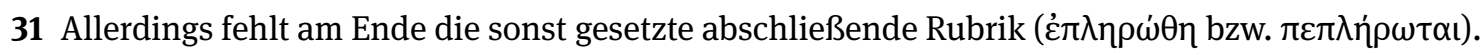

32 Fragmente einer sonst nicht überlieferten anhomöischen Erklärung. Einleitung $\S 16$ und Zitat $\S$ $17-22$.

33 Unterschriften unter der theologischen Erklärung der Synode von Sirmium 359. Einleitung § 24 und $\S 25-29$. 
blem bietet außerdem die Rubrik, die in der Handschrift zwischen den beiden zitierten Dokumenten steht. ${ }^{34}$

\section{Der Aufbau von Synodalschreiben}

Bevor ich mich dem Brief der Synode von Ankyra selbst zuwende, möchte ich zuerst einmal untersuchen, wie ein Synodalschreiben eigentlich aufgebaut ist. Das erscheint deswegen als weiterführend, weil es dadurch - bei allen zu machenden Einschränkungen auf Grund der sehr kleinen von mir herangezogenen Beispielauswahl - besser möglich ist, die Eigenheiten des Briefes der Synode von Ankyra zu erfassen. Als Beispiele greife ich zum Vergleich zwei der Texte heraus, auf die sich die Synode von Ankyra auch selbst bezieht, sie also kannte und positiv rezipierte, ${ }^{35}$ so dass die Parallelen wie Differenzen als besonders signifikant anzusehen sind.

Es handelt sich um die Dokumente der »östlichen « Synode von Serdika ${ }^{36}$ und um die »Ekthesis makrostichos « ${ }^{37}$. Die anderen im Brief erwähnten Synoden ${ }^{38}$ eignen sich deshalb weniger zum Vergleich, weil wir von den entsprechenden Synoden nur eine sehr selektive Auswahl an Dokumenten überliefert haben, die mit dem Überlieferungsbestand der Synode von Ankyra nicht vergleichbar sind, und werden daher nicht behandelt.

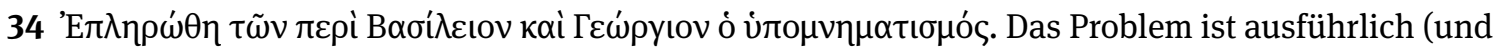
in Auseinandersetzung mit der übrigen Literatur) in DelCogliano, The Literary Corpus of George of Laodicea, 164-169 diskutiert. Die Rubrik bleibt problematisch, und es ist zumindest die zweifelnde Frage zu stellen, ob zum einen eigentlich wirklich so sicher davon auszugehen ist, dass die Rubriken generell auf Epiphanius selbst zurückgehen und nicht auf einen späteren Redaktor (vergleichbar der Anakephalaiosis), oder ob zum anderen im Laufe der Überlieferung die Rubrik durch einen Fehler nicht einfach vom Ende des Traktates Georgs an diese Stelle verrutscht ist (vgl. oben Anm. 31), da Epiphanius im Traktat Georgs ja wahrscheinlich den in $\S 4$ des Briefes der Synode angekündigten Brief Georgs erkannt hat und diesen dann vergleichbar zu den am Ende des Traktates zitierten Texten einfach ohne weitere Rubrik angehängt hätte. Die Frage wird sich angesichts der schlechten Überlieferung des Epiphanius nicht abschließend lösen lassen.

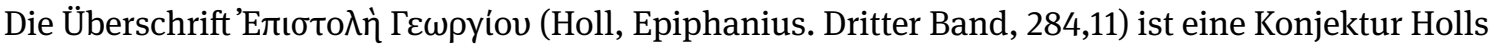
ohne jeglichen Anhaltspunkt in der Überlieferung und ist daher auf jeden Fall zurückzuweisen.

35 Vgl. Dok. 55,2.4.

36 Dok. 43.11-13, AW III 3, 250-279.

37 Dok. 44, AW III 3, 280-287.

38 Die Synode von Konstantinopel 336[?] (Dok. 40), die Synode von Antiochien 341 [2. antiochenische Formel] (Dok. 41.4) und die Synode von Sirmium 351 (Dok. 47). 


\subsection{Die Dokumente der »östlichen« Synode von Serdika (Dok. 43.11-13)}

Von der »östlichen « Synode von Serdika sind uns bei Hilarius (coll. antiar. A IV 1-3) drei Dokumente im Zusammenhang überliefert. Er allein bietet neben der Ekthesis auch den Rundbrief und die Unterschriftenliste, während er selbst in seiner Schrift syn. 34 ebenso wie die Sammlungen Codex Veronensis LX und Codex Parisinus syr. 62 nur die Ekthesis der Synode überliefern. ${ }^{39}$

Bei den Texten der »östlichen« Synode von Serdika handelt sich um ein »Dokumentenkonvolut«, bestehend aus Rundbrief, Ekthesis und Unterschriftenliste:

Der (lange) Rundbrief (Dok. 43.11) ist folgendermaßen aufgebaut: Auf den Gruß, der Adressaten und Verfasser nennt $(\S 1)$, beginnt das Schreiben mit einem Bekenntnis zur Tradition (§ 2), die für die Verfasser die Grundlage ihres Handelns darstellt. Nach zwei ausführlichen historischen Rückblicken auf die Verurteilung Markells von Ankyra (§ 3-6) und auf die Verurteilung des Athanasius von Alexandrien (§ 7-14) werden in einem abschließenden, ebenfalls umfangreichen Teil (§ 15-29) die Vorgänge auf der Synode von Serdika geschildert und am Ende die Verurteilungen durch die Synode sowie die Abfassung einer an die Tradition anschließenden Ekthesis mitgeteilt, die von den Adressaten unterschrieben werden soll. Ein Schlussgruß fehlt.

Die Ekthesis (Dok. 43.12) zitiert (ohne das anzugeben) nach einem Präskript mit der Nennung der Synode und der auf ihr repräsentierten Provinzen (§ 1) in § 2-4 die 341 auf einer Synode in Antiochien verabschiedete Ekthesis (Dok. 42) samt ihren (schon aus dem Nicaenum übernommenen) namentlich nicht genannte Häresien verurteilenden Anathematismen und fügt diesen deutlich markiert ${ }^{40}$ im selben Stil ${ }^{41}$ noch weitere hinzu. Dem schließt sich die Liste (Dok. 43.13) mit 73 angeführten Unterschriften, bestehend aus Name und Bischofsort, an.

39 Die beiden Handschriften Parisinus syr. 62 und Codex Veronensis LX schließen an die Ekthesis den Text über den Osterzyklus (Paris. syr. 62 bietet nur die Rubrik und lässt entsprechend die vom Veron. LX außerdem noch überlieferten Texte des Briefes des Ossius und Protogenes, Dok. 43.6, sowie des Rundbriefes und der Ekthesis der "westlichen « Synode, Dok. 43.1 und 43.2, aus) und die Kanones der "westlichen « (!) Synode von Serdika an, vgl. dazu auch Camplani, Fourth-Century Synods in Latin and Syriac, v.a. 64. Passend zu den Kanones der »westlichen « Synode bieten die beiden Sammlungen Veronensis LX und Parisinus syr. 62 einen im Sinne der nizänischen Orthodoxie korrigierten Text des letzten Anathematismus (AW III 3, 275,10 f. vgl. auch Camplani, Fourth-Century Synods in Latin and Syriac, 67-69). Außerdem wird in den drei Zeugen, die ausschließlich die Ekthesis anführen, dieser auch ein eigenes Präskript (Dok. 43.12,1) vorangestellt, während Hilarius in coll. antiar. A IV 2 dieses kürzt und an seiner Stelle einen überleitenden Satz bietet: Est autem fides nostra talis. Ein vergleichbarer Fall liegt in dem im Paris. syr. 62 überlieferten Präskript zu den Kanones (vgl. Schulthess, Die syrischen Kanones, 168,4-11) mit der Angabe der Synode und den beteiligten Provinzen (mit minimalen Abweichungen zur Theodoret-Überlieferung in Dok. 43.1,1 App. zu Z. 1 f.) vor.

40 Vgl. AW III 3, 275,5-12: Similiter et eos ... anathematizat sancta et catholica ecclesia.

41 Vgl. dagegen die Formulierung der Anathematismen im Schreiben der Synode von Antiochien, unten Anm. 63. 


\subsection{Ekthesis Makrostichos (Dok. 44)}

Ein etwas anderes Bild bietet sich im Fall der »Ekthesis makrostichos«, die bei Athanasius in seiner Schrift De synodis und von diesem abhängig bei Sokrates in seiner Kirchengeschichte überliefert ist. Sehr wahrscheinlich dadurch bedingt ${ }^{42}$ finden sich hier weder ein Präskript noch eine Unterschriftenliste, sondern einzig die Ekthesis dieser antiochenischen Synode des Jahres 344. ${ }^{43}$

Die Ekthesis setzt mit einem (freilich nicht als solchem markierten) Zitat der Ekthesis der »östlichen« Synode von Serdika ein (§ 1-4). Daran anschließend werden die

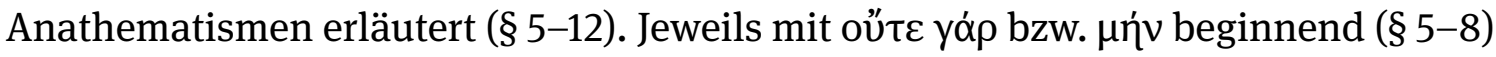
werden die beiden noch auf das Nizaenum zurückgehenden Anathematismen erläutert, ${ }^{44}$, anschließend die serdicensischen, ${ }^{45}$ wobei in $\S 8$ mit Paulus von Samosata der Urheber der Häresie namentlich benannt wird. Anschließend wird mit $\beta \delta \varepsilon \lambda v \sigma \sigma o ́ \mu \varepsilon \theta \alpha$ (wir verabscheuen) ein starker Einschnitt markiert, und die eigentlichen Gegner der Verfasser des Textes werden deutlich: Markell und Photin (§ 9-10), ehe in § 11 gegen Sabellius und in $\S 12$ (wieder ohne Namensnennung) gegen Aëtius argumentiert wird.

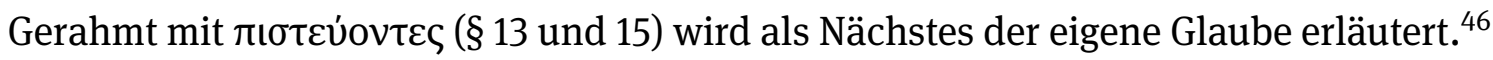

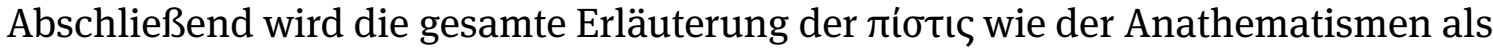
notwendig festgestellt (§ 16).

\section{Aufbau und Argumentationsstrukturen des Briefes der Synode von Ankyra}

Beide als Vergleichstexte angeführten Ektheseis zitieren also in der Hauptsache einen bereits vorliegenden Bekenntnistext, der anschließend aktualisierend, sei es durch weitere Anathematismen, sei es durch erläuternde Kommentare, erweitert wird. Von diesem Schema der Rezeption der Tradition durch Zitation weicht der Brief der Synode von Ankyra signifikant ab. ${ }^{47}$

42 Athanasius hatte an historischen Daten kein Interesse, ihm geht es beim Zitat der Ekthesis um den Beweis seiner These, dass die »Arianer « in kurzen Abständen immer neue Bekenntnistexte beschlossen haben, vgl. den einleitenden Satz zur Ekthesis in syn. 26,1 und vor allem den durch syn. 21,1 und syn. 32,1 gebildeten Rahmen des gesamten, die diversen Bekenntnisse zitierenden Abschnittes von De Synodis.

43 Vgl. die Einleitung zum Text in AW III 3, 281.

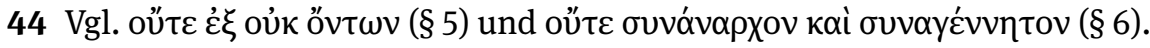

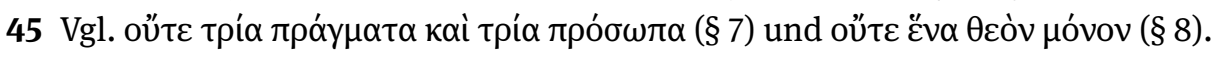

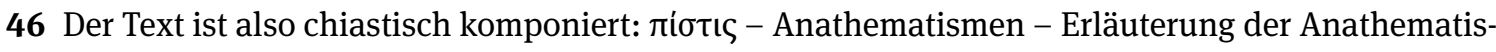

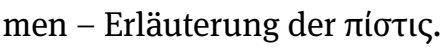

47 Vgl. allerdings unten S. 200 zu den Parallelen mit dem Synodalschreiben der »östlichen« Synode von Serdika. 
Das Schreiben der Synode von Ankyra wird traditionell in drei Teile gegliedert: ${ }^{48}$ Einleitung, Darlegung der homöusianischen Lehre und Anathematismen. Demgegenüber möchte ich hier für eine Vier- oder, wenn man die Unterschriften als eigenen Abschnitt zählt, sogar für eine Fünfteilung des Briefes plädieren:

\begin{tabular}{|c|c|}
\hline$\S 1-5$ & Einleitung \\
\hline$\S 6-7$ & 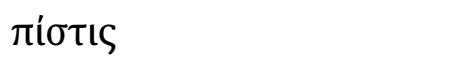 \\
\hline$\S 8-25$ & Homöusianischer Traktat \\
\hline$\S 26$ & 19 Anathematismen \\
\hline 27 & Unterschriften \\
\hline
\end{tabular}

Neben den offensichtlichen Gliederungseinschnitten am Ende von $\S 5$ (Einleitung), mit $\S 26$ (Anathematismen) und mit $\S 27$ (Unterschriften) lässt sich auch im theologischen, argumentativen Teil noch ein Gliederungseinschnitt feststellen, nämlich zwischen der Darlegung des eigenen Glaubens in $\S 6$ und 7 und dem darauf folgenden theologischen Traktat ( 8 8-25). Diese Unterteilung wird dadurch deutlich, dass die Verfasser des Briefes in $\S 6$ und 7 noch in der 1. Person von ihrem Glauben sprechen, ${ }^{49}$ während sie $\mathrm{ab} \S 8$ sukzessive in eine deskriptive und argumentative Diktion übergehen.

Ist der theologische Traktat in § 8-25 nach vorne an die Pistis durch einen sukzessiven Übergang gut angeschlossen, so bricht sie doch am Ende an der Schnittstelle zu den Anathematismen trotz des zusammenfassenden Charakters von $\S 25$ insofern abrupt ab, als sich die Anathematismen (§ 26) ohne Übergang oder Einleitung direkt anschließen. Außerdem fällt auf, dass die Anathematismen die Argumente des Traktates auch nicht Punkt für Punkt in derselben Reihenfolge aufnehmen, sondern einem eigenen Duktus folgen. ${ }^{50}$ Schließlich fehlt der Briefschluss mit einem Schlussgruß, der wahrscheinlich bereits bei der redaktionellen Bearbeitung des Textes durch Epiphanius entfernt wurde. Unklar ist, ob er ursprünglich vor oder (wahrscheinlicher) nach den Anathematismen zu stehen kam, ob also die Anathematismen einen Anhang zum Brief darstellen oder vielmehr integraler Teil des Briefes sind. Dementsprechendes gilt für die heute nur noch summarische, also ebenfalls redaktionell bearbeitete Unterschriftenliste. ${ }^{51}$ Außerdem bleibt die Frage, wo eigentlich der in § 4 genannte Anhang mit dem Brief Georgs von Laodizäa zu stehen kam, ob vor oder (wahrscheinlicher, d. h. als eigenes, dem Brief beigelegtes Blatt) nach den Anathematismen und der Unterschriftenliste.

\footnotetext{
48 Vgl. Kopecek, A history of Neo-Arianism, 156; Löhr, Entstehung der homöischen und homöusianischen Kirchenparteien, 63.

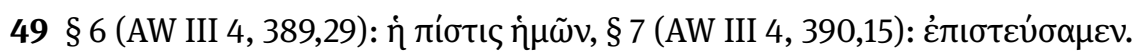

50 Vgl. die Tabelle auf S. 202.

51 Es ist sehr wahrscheinlich, dass der Schlussgruß des Briefes im Zuge der redaktionellen Kürzung der Unterschriftenliste in $\S 27$ weggefallen ist.
} 
Im einleitenden Teil des Briefes (§ 1-5) erläutern die Verfasser nach dem formalen Briefgruß (§ 1) ${ }^{52}$ Anlass und Ziel ihres Schreibens (§ 2-4): Anlass ihres Schreibens ist das Aufkommen einer neuen Häresie, nämlich der »Anhomöer« (§ 3), und Ziel ihres Schreibens ist die Unterstützung der Einheitspolitik des Kaisers Constantius und dadurch die Zurückdrängung der neuen Häresie (§ 4). Ihre Intention und vor allem auch ihr Selbstverständnis drücken sie dabei deutlich aus: ${ }^{53}$

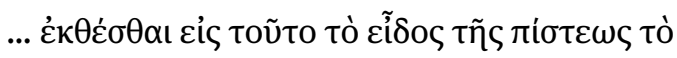

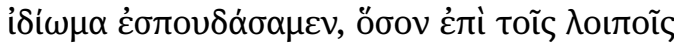

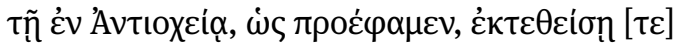

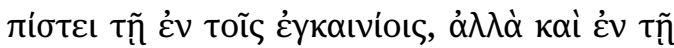

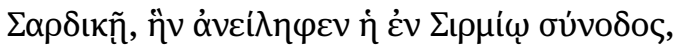

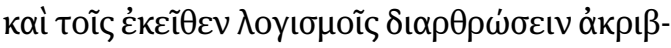

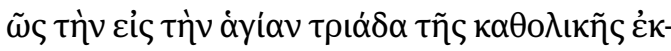

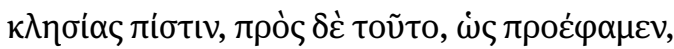

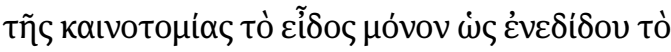
$\pi v \varepsilon \tilde{u} \mu \alpha$ ن் $\alpha$
... und uns bemüht, in dieser Form das Wesentliche des Glaubens darzulegen, der wie gesagt im übrigen auf der Kirchweihsynode in Antiochien festgelegt worden ist, aber auch auf der Synode in Serdica, den die Synode von Sirmium übernahm, und mit den von dort ausgehenden Erwägungen den Glauben der katholischen Kirche an die heilige Trinität genau gegliedert darzulegen, wobei wir dazu, wie wir gesagt haben, auf diese Art der Neuerung nur so geantwortet haben, wie es der Geist eingab.

Die Synodalen greifen also auf die Bekenntnisse von Antiochien (Dok. 41.4), von Serdika »Ost « (Dok. 43.11-13), von Sirmium (Dok. 47) und - freilich etwas kryptisch - auf die Ekthesis makrostichos (Dok. 44) zurück, ${ }^{54}$ allerdings ohne sie zu zitieren. ${ }^{55}$ Damit stehen sie im deutlichen Gegensatz zu den von ihnen referenzierten und von mir bereits etwas näher betrachteten Bekenntnissen, weil diese jeweils das Bekenntnis, auf das sie zurückgreifen, wörtlich zitieren und dann gegebenenfalls (aktualisierend) ergänzen. Die in Ankyra versammelten Bischöfe führen überhaupt keinen vollständigen Bekenntnistext an, so dass wir es nicht mit einer Ekthesis im strengen Sinne zu tun

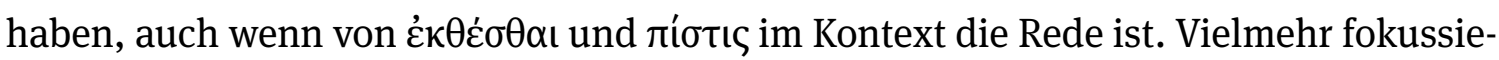

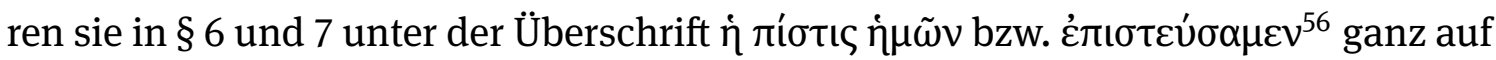
die aktuell strittigen Punkte, um sich gegen die durch die Heterousianer eingeführten Neuerungen zur Wehr zu setzen - denn das ist am Ende des Satzes gemeint, nicht dass die Verfasser die eigenen Darlegungen als Neuerungen verstehen! Vielmehr reihen sie sich mit der Aufzählung der Synoden in einen langen Traditionszusammenhang ein. ${ }^{57}$

52 Das Brief ist ein synodales Rundschreiben, dessen in die Provinz Phoenice abgesandtes Exemplar Epiphanius vorgelegen hat.

53 Dok. 55,4 (AW III 4, 388,34-389,11). Instruktiv ist der Vergleich mit der dieselbe Funktion erfüllenden Formulierung der Ekthesis makrostichos (Dok. 44,16), vgl. oben Abschnitt 3.2.

$54 \mathrm{Vgl}$. auch die Aufzählung in § 2.

55 Der Rückgriff auf die Tradition ist aber durchaus vergleichbar mit Dok. 43.11,2.

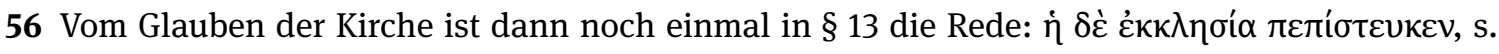
folgende Anmerkung.

57 Auf diesen Traditionszusammenhang wird dann auch im Traktat verwiesen, allerdings ist der Referenzrahmen dort nicht mehr eine synodale Tradition, sondern die heilige Schrift selbst (§13 f.16-25). 
Damit - wie auch durch den Rückgriff auf den Taufbefehl $(55,6)^{58}$ - schließen sie sich strukturell eher an die Ekthesis der Synode von Sirmium des vorangegangenen Jahres an (Dok. 51), die im Text selbst aber mit keinem Wort erwähnt wird. ${ }^{59}$

$\S 5$ schließt die Einleitung mit einem Appell an die direkt angesprochenen Adressaten unter Bezugnahme auf den apostolischen Glauben zur bekräftigenden Unterschrift

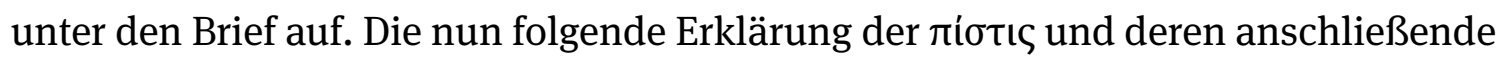
Erläuterung sind somit die programmatische Darlegung der Theologie, der sich die Adressaten anschließen sollen.

In $\S 6 \mathrm{f}$. wird ausgehend vom Taufbefehl anhomöische Diktion zurückgewiesen und an ihrer Stelle die Rede von der ö $\mu$ oเ $\alpha$ oủoí $\alpha$ des Sohnes bzw. vom Sohn, der dem Vater ő $\mu$ oเoৎ ist, eingeführt. ${ }^{60}$ Sprachlich sehr massiv wird außerdem die Bezeichnung

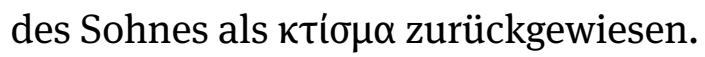

Mit $\delta$ เó eingeleitet folgt dann der lange Abschnitt § 8-25, der vom Umfang ungefähr die Hälfte des Schreibens ausmacht und sich vom »Wir«-Teil zu Beginn und von den Anathematismen am Ende stilistisch deutlich abhebt. Dieser Abschnitt wird in der Forschung meist nur inhaltlich als »Darlegung der homöusianischen Lehre« beschrieben und als integraler Bestandteil des Briefes verstanden. Ich habe ihn demgegenüber als theologischen bzw. homöusianischen Traktat betitelt. Dahinter steht die Vermutung, dass es sich bei ihm (trotz einiger Spuren von Mündlichkeit) um ein von Basilius von Ankyra für die Synode vorbereitetes Schriftstück handelt, das in einer redaktionellen Phase der Paraphierung des Synodalschreibens in dieses eingepasst wurde.

In ihm wird als theologische Denkfigur gegen die zurückgewiesenen Lehren der Heterousianer auf der einen (§ 16-22) und der Homousianer auf der anderen Seite (§ 23-25) unter immer wieder betontem Ausschluss jeglichen körperlichen Verständnisses

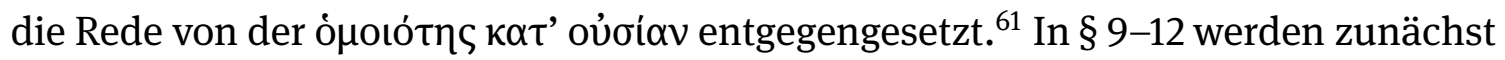
vier Einwände vorgestellt, wobei die ersten drei (§ 9-11) jeweils mit der Konjunktion عi eingeleitet werden. Diesen Einwänden wird in einem nächsten Schritt die Position der kirchlichen Tradition entgegengesetzt (§ 13 f.): Gott ist Schöpfer der Geschöpfe

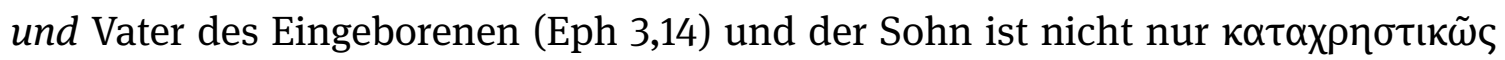
und ó $\mu$ ovv́ $\mu \omega \varsigma$ Sohn, d. h. die in den vorangehenden Einwänden in der gegnerischen Argumentation vorgebrachten biblische Verse Hi 38,28, Jes 1,2 und Joh 1,12 sind nicht mit dem Verhältnis Vater - Sohn vergleichbar. In $\S 15$ wird - wiederum durch عi eingeleitet - ein weiterer Einwand vorgebracht: Die Rede vom Sohn impliziere logisch

58 Dieser ist aber auch schon in der 2. antiochenischen Formel der Ausgangspunkt der Bekenntnisformulierung.

59 Einzig die Nennung Illyriens in $\S 4$ und die damit verbundene Anspielung auf Mitteilungen über neuerlichen Ärger lassen an dieses Dokument denken.

60 AW III 4, 390,11.13.

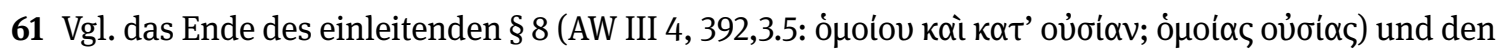

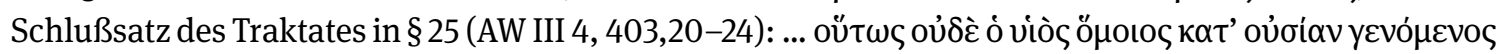

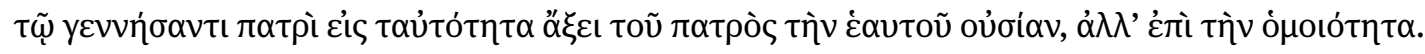




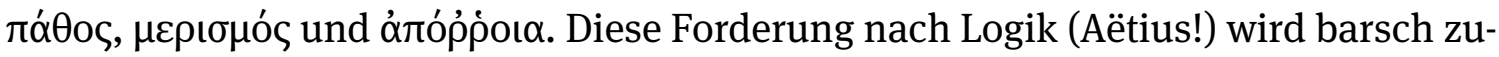
rückgewiesen und unter Berufung auf Paulus (1Kor 1,17.20; 2,1) der Logik der Glaube als adäquater Maßstab theologischen Denkens entgegengesetzt. Die daran anschließende Gegenargumentation ist daher ein ausführlicher Schriftbeweis (§ 16-22) in drei bzw.

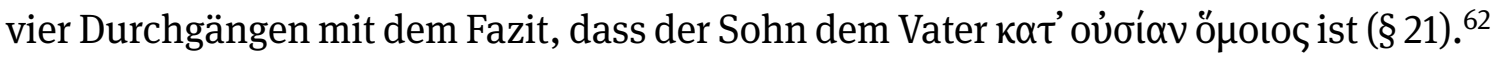

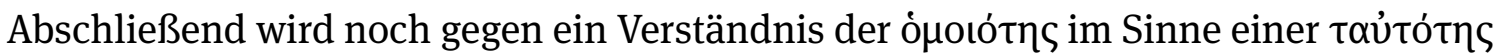
argumentiert, wie sie den Nizänern vorgeworfen wurde. Das Hauptaugenmerk der Argumentation ist eindeutig auf die »Anhomöer « gerichtet, eine Abgrenzung zu den »Nizänern « findet zwar statt, steht aber eher am Rande.

Demgegenüber wenden sich die Anathematismen (§ 26) in ausgeglichenerem Maße gegen beide Gruppen: ${ }^{63}$

1. Zusammenfassung (gegen Rede von Schöpfer und Geschöpf anstelle von Vater und Sohn)

2.-9. Vier Anathematismen-Paare gegen Markell (Identität) bzw. »Anhomöer « (Ungleichheit)

2.-3. bezüglich бo甲ía (vgl. § 16-18)

4. -5. bezüglich $\lambda$ óyos (vgl. § 21)

6. -7. bezüglich عik

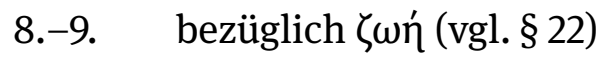

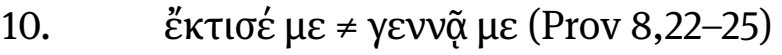

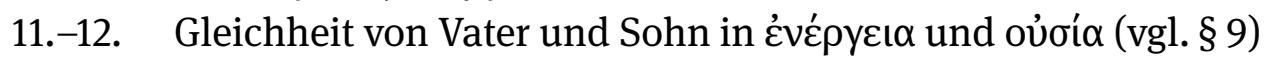

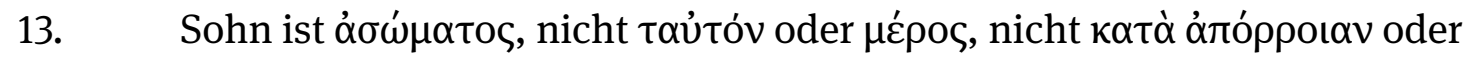

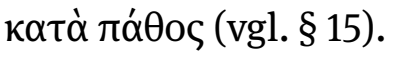

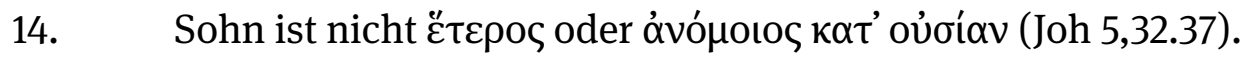

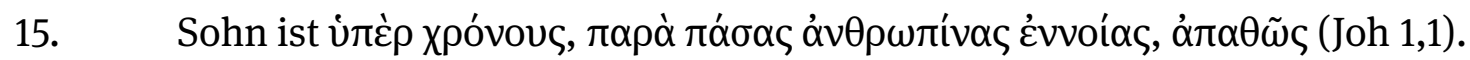

16. Vater ist nicht älter, Sohn nicht jünger der Zeit nach.

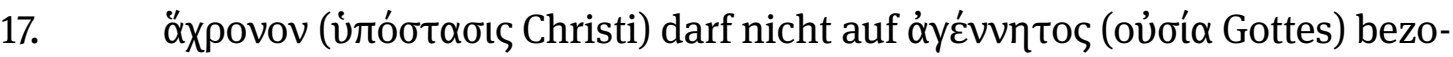
gen werden.

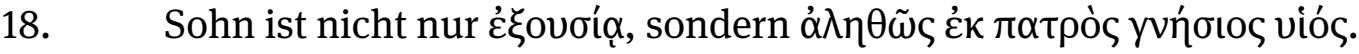

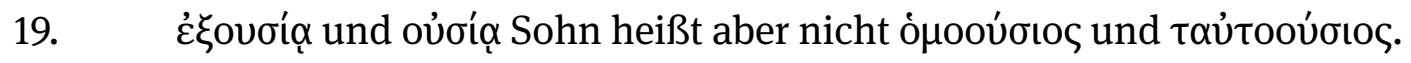

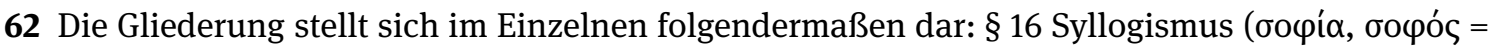

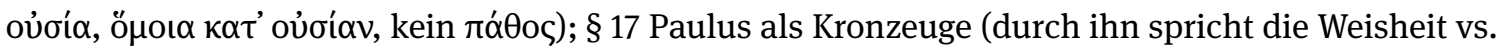

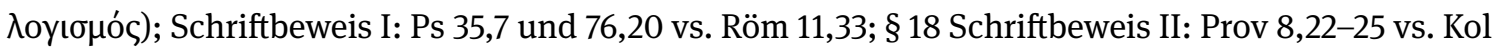
1,15; § 19 Schlussfolgerung; § 20-21 dafür ist nicht nur Paulus, sondern auch Johannes Zeuge, daher Schriftbeweis III: Prov 8,22-25 vs. Joh 1,1.14 und 5,19; § 22 Weiterer Beweis aus der Schrift: Joh 5,26.

63 Die Anathematismen 6-17 werden von der Synode von Sirmium im Jahr 358 rezipiert, vgl. Dok. 56.3. In der Art der Formulierung schließen sich diese Anathematismen an die ebenfalls als Referenz aufge-

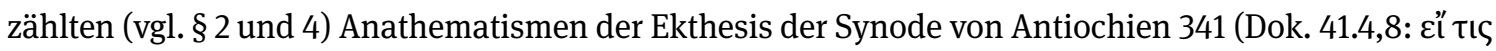

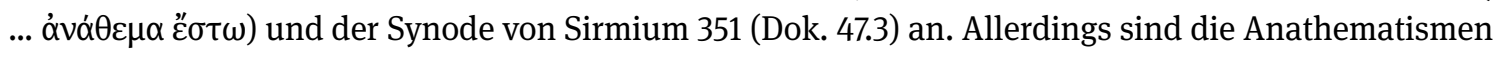
der Synode von Ankyra jeweils viel umfangreicher. 
An die Anathematismen ist abschließend die (gekürzte) Unterschriftenliste angefügt.

\section{Zusammenfassung: Die Synode von Ankyra}

Vor allem in den drei Teilen des homöusianischen Traktates, der Anathematismen und der Unterschriften stehen uns drei Quellen zur Verfügung, die uns einen (freilich nur hypothetischen) Einblick in den Ablauf der Synode gewähren können, über den wir sonst überhaupt nicht unterrichtet sind. ${ }^{64}$ Denn die viel spätere Darstellung beim Kirchenhistoriker Sozomenus berichtet nur vom Faktum der Synode und von der Delegation an den Hof des Kaisers in Sirmium im Anschluss an die Synode, ${ }^{65}$ von der wir wiederum aus dem Rundbrief der Synode, die unsere einzige Primärquelle darstellt, überhaupt nichts erfahren.

Ich hatte oben die Vermutung geäußert, dass es sich bei dem »homöusianischen Traktat« um eine der Synode schriftlich vorliegende theologische Auseinandersetzung des Basilius von Ankyra v. a. mit der drohenden neuen Häresie der »Anhomöer« handelt. Trifft dies zu, so ist es naheliegend, dass dieser Text nicht nur in den Synodalbrief eingefügt worden ist, sondern vielmehr dafür abgefasst wurde, um während der synodalen Verhandlungen eine Diskussionsgrundlage $\mathrm{zu}$ bieten, und so auch auf der Synode verwendet wurde. Auf der Synode von Ankyra wurden ja nicht nur altbekannte Theologumena wiederholt und ausgetretene theologische Wege beschritten, sondern angesichts einer ganz neuen Herausforderung neue theologische Lösungen erarbeitet. Es ist schwer vorstellbar, dass dies ohne Diskussion abgelaufen wäre. Dafür spricht auch, dass in den wahrscheinlich am Ende der Diskussionen verabschiedeten Anathematismen die Akzente zwar kompatibel zum theologischen Traktat, aber durchaus anders gesetzt werden.

Die Unterschriftenliste schließlich dürfte ganz am Ende der Synode stehen, da sie direkt zum Brief der Synode gehören und gewissermaßen das vorwegnehmen, wozu die Absender des Briefes ihre Adressaten in $\S 5$ auffordern.

Damit wären auf Grundlage des Rundbriefes drei Phasen im Ablauf der Synode auszumachen:

1. Theologische Positionsbildung auf Basis des Traktates des Basilius

2. Abfassung der Anathematismen

3. Abfassung des Synodalschreibens und seine Unterzeichnung

64 Der Einleitungsteil mit seinem Rückblick auf die vorangegangenen Synoden macht nicht nur das Selbstverständnis der Synode deutlich, sondern verweist auch auf die Vorgeschichte und damit die Beweggründe, die zur Synode geführt haben.

65 Soz., h. e. IV 13. 


\section{Ausblick}

In meinem Beitrag habe ich versucht $\mathrm{zu}$ zeigen, mit welchen überlieferungsgeschichtlichen und inhaltlichen Problemen das Schreiben der Synode von Ankyra aus dem Jahr 358 belastet ist und wie überlieferungsgeschichtliche und textkritische Probleme mit inhaltlichen Fragestellungen verwoben sind. Insofern kann dieses Dokument als Exempel für jeden einzelnen (Synodal-)Text aus dem Kontext des »arianischen« Streites dienen, wenn es um die Frage geht, wie überhaupt die Dokumente einer Synode auf uns gekommen sind und wie und in welcher Form (und das heißt vor allem auch in welchem Zuschnitt und mit welchen redaktionellen Eingriffen) sie in die kirchengeschichtlichen und polemisch-apologetischen Werke Eingang gefunden haben und rezipiert worden sind.

\section{Literatur}

Brennecke, Hanns Christof/Uta Heil/Annette von Stockhausen/Angelika Wintjes, Athanasius Werke. Band III. Teil 1: Dokumente zur Geschichte des Arianischen Streits 318-328. 3. Lfg.: Bis zur Ekthesis Makrostichos, Berlin/New York, 2007.

Brennecke, Hanns Christof/Annette von Stockhausen/Christian Müller/Uta Heil/Angelika Wintjes, Athanasius Werke. Band III. Teil 1: Dokumente zur Geschichte des Arianischen Streits 318-328. 4. Lfg.: Bis zum Tomus ad Antiochenos (362), Berlin/Boston, 2014.

Camplani, Alberto, Fourth-Century Synods in Latin and Syriac Canonical Collections and their Preservation in the Antiochene Archives (Serdica 343 CE - Antioch 325 CE), in: Cultures in Contact. Transfer of Knowledge in the Mediterranean Context. Selected Papers, hrsg. von Juan Pedro Monferrer Sala/Sofía Torallas Tovar, Syro-Arabica 1, Cordoba/Beirut, 2013, 61-72.

Cornarius, Janus, D. Epiphanii episcopi Constantiae Cypri, Contra octoaginta haereses opus, Basel, 1543.

DelCogliano, Mark, The Literary Corpus of George of Laodicea, in: Vigiliae Christianae 65 (2011), 150-169.

Dummer, Jürgen/Christoph Markschies, Philipp Melanchthons Randbemerkungen zu Epiphanius im Jenensis Ms. Bos. f. 1, in: Epiphanius I: Ancoratus und Panarion haer. 1-33, hrsg. von Karl Holl/Marc Bergermann/Christian-Friedrich Collatz, Zweite, wesentlich erweiterte Auflage, Die griechischen christlichen Schriftsteller der ersten Jahrhunderte. Neue Folge 10, Berlin/Boston, 2013, 795-818.

Fairbairn, Donald, The Synod of Ancyra (358) and the Question of the Son's Creaturehood, in: Journal of Theological Studies 64 (2013), 111-136.

Gummerus, Jaakko, Die homöusianische Partei bis zum Tode des Konstantius. Ein Beitrag zur Geschichte des arianischen Streites in den Jahren 356-361, Leipzig, 1900.

Hanson, Richard P. C., The Search for the Christian Doctrine of God. The Arian Controversy 318-381, Edinburgh, 1988.

Holl, Karl, Die handschriftliche Überlieferung des Epiphanius: (Ancoratus und Panarion), Texte und Untersuchungen zur Geschichte der altchristlichen Literatur 36/2, Leipzig, 1910.

- Epiphanius. Dritter Band: Panarion haer. 65-80. De fide, Die griechischen christlichen Schriftsteller der ersten Jahrhunderte 37, Berlin, 1933. 
Holl, Karl/Jürgen Dummer, Epiphanius. Dritter Band: Panarion haer. 65-80. De fide, 2. Aufl., Die griechischen christlichen Schriftsteller der ersten Jahrhunderte. Neue Folge 3, Berlin, 1985.

Jacobs, Andrew S., Epiphanius of Cyprus. A cultural biography of late antiquity, Oakland, California, 2016.

Kim, Young Richard, Epiphanius of Cyprus. Imagining an orthodox world, Ann Arbor, 2015.

Kopecek, Thomas A., A history of Neo-Arianism, Patristic monograph series 8, Cambridge, Mass., 1979.

Lienhard, Joseph T., The Epistle of the Synod of Ancyra 358. A Reconsideration, in: Arianism. Historical and theological reassessments. Papers from the Ninth International Conference on Patristic Studies, September 5-10, 1983, Oxford, England, hrsg. von Robert Gregg, Patristic monograph series 11, Cambridge, Mass., 1985, 313-320.

Löhr, Winrich Alfried, A Sense of Tradition: The Homoiousian Church Party, in: Arianism After Arius. Essays on the Development of the Fourth Century Trinitarian Conflicts, hrsg. von Michel Barnes/Daniel H. Williams, Edinburgh, 1993, 81-100.

- Die Entstehung der homöischen und homöusianischen Kirchenparteien. Studien zur Synodalgeschichte des 4. Jahrhunderts, Bonner Beiträge zur Kirchen- und Theologiegeschichte 2, Bonn, 1986.

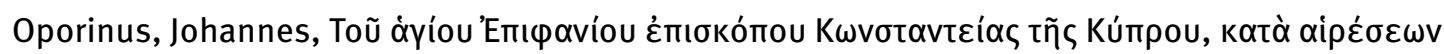
óүठоńкоvта... D. Epiphanii episcopi Constantiae Cypri, Contra octoginta haereses ... Basel, 1544.

Petavius, Dionysius, Hrsg., Epiphanii Opera omnia in duos tomos distributa, Paris, 1622.

Schulthess, Friedrich, Die syrischen Kanones der Synoden von Nicaea bis Chalcedon nebst einigen zugehörigen Dokumenten, Abhandlungen der königlichen Gesellschaft der Wissenschaften zu Göttingen. Philologisch-historische Klasse. Neue Folge 10,2, Berlin, 1908.

Steenson, Jeffrey N., Basil of Ancyra and the Course of Nicene Orthodoxy, London, 1984.

Stockhausen, Annette von, Katalog der griechischen Handschriften im Besitz der Thüringer Universitäts- und Landesbibliothek Jena, in: Byzantinische Zeitschrift 94 (2001), 684-701. 